\title{
Artículos
}

\section{Objetivos priorizados y metodologías más utilizadas por los extensionistas rurales argentinos*}

Cómo citar este artículo: Landini, F. P., y Beramendi, M. R. (2020). Objetivos priorizados y metodologías más utilizadas por los extensionistas rurales argentinos. Cuadernos de Desarrollo Rural, I7. https://doi.org/ı0.III44/Javeriana.cdri7.opmu

Fernando Pablo Landini ${ }^{\text {a }}$

Universidad de la Cuenca del Plata, Argentina

landini_fer@hotmail.com

ORCID: https://orcid.org/0000-0002-5322-292I

Maite Regina Beramendi

Universidad de Buenos Aires, Argentina

ORCID: https://orcid.org/0000-0002-6113-5529

DOI: https://doi.org/I0.III44/Javeriana.cdrı7.opmu

Recibido: 07 Mayo 2019 I Aceptado: 27 Junio 2020 I Publicación: 20 Diciembre 2020

\section{Resumen:}

En el presente trabajo, se evaluaron los objetivos priorizados y las estrategias metodológicas más utilizadas por los extensionistas rurales de dos principales instituciones de extensión en Argentina. Para lo anterior, se formuló un cuestionario que fue respondido por 593 extensionistas. Los objetivos más mencionados son la creación y fortalecimiento de organizaciones de productores, el apoyo al acceso a servicios básicos y el fortalecimiento de las estrategias productivas a través de la financiación de pequeños proyectos. Las estrategias metodológicas grupales son las más utilizadas. Los objetivos y las metodologías difieren, fundamentalmente, según la organización de pertenencia, años de experiencia y título académico de los extensionistas.

Palabras clave: extensión rural, objetivos, metodologías, Argentina.

a Autor de correspondencia. Correo electrónico: landini_fer@hotmail.com 


\section{Introducción}

La extensión rural es una práctica social compleja y diversa, que involucra una multiplicidad de metodologías, abordajes y objetivos (Sulaiman y Davis, 20I2). En este sentido, es frecuente que las definiciones de extensión rural hagan énfasis en ciertas dimensiones y no en otras. En este trabajo, se emplea una definición orientativa y genérica de extensión rural que hace referencia a "las diferentes actividades de suministro de información y de asesoramiento pertinentes que solicitan los agricultores y otros actores en los sistemas agroalimentarios y el desarrollo rural” (Christoplos, 2010, p. 2).

En un sentido clásico, esta diversidad de metodologías, abordajes y objetivos hace referencia al contraste entre una extensión rural difusionista, orientada a la modernización productiva y a la transferencia tecnológica (Rogers, 1962), y a una extensión rural pensada como proceso educativo de diálogo, orientado a la concientización y a la construcción conjunta de conocimientos superadores (Freire, 1973). No obstante, una mirada atenta permite observar una diversidad de prácticas, metodologías y objetivos mucho más amplia. Así, junto a la clásica propuesta de transferencia de tecnologías y de modernización productiva, diferentes autores han señalado el papel de la extensión rural en la creación y fortalecimiento de organizaciones de productores (Landini, 2016a), la protección y el manejo de recursos naturales (Abdu-Raheem, 20I4), el desarrollo de la capacidad emprendedora de los agricultores (Stevens, 2017), el apoyo a la comercialización y a la integración en los mercados (Christoplos, 2010), el aporte a la seguridad alimentaria (Ardila, 2010), entre otros.

En cuanto a la diversidad metodológica, puede identificarse un primer contraste entre abordajes individuales, centrados en el asesoramiento técnico, y abordajes grupales, generalmente caracterizados por procesos participativos o dinámicas de aprendizaje conjuntas (Acunzo et al., 20I6; Rossi, 20II). No obstante, también es necesario señalar la importancia que tradicionalmente ha tenido el uso de medios de comunicación, tales como la radio y la televisión, para transmitir información agropecuaria, lo que se ha reforzado en los últimos años con las redes sociales, internet y los teléfonos inteligentes (Galindo González et al., 200I; González Tena et al., 20I5). Por último, en el contexto de un abordaje territorial del desarrollo rural, también puede considerarse la articulación y coordinación interinstitucional (Torrado Porto y Catullo, 2017) como una estrategia de extensión rural por derecho propio. Desde una perspectiva de desarrollo territorial, se reconoce la importancia de tomar conciencia de la heterogeneidad social de los espacios rurales y de facilitar la articulación entre una multiplicidad de actores para, de esta forma, impulsar procesos de transformación institucional y productiva (Schejtman y Berdegué, 2004). En este marco, es importante señalar que los autores no consideran la utilización de estrategias o metodologías de extensión individuales, grupales, de coordinación institucional o de uso de medios masivos de comunicación como opciones contrapuestas, sino más bien como complementarias (Acunzo et al., 20I6; Kingiri y Nderitu, 20I4).

Ahora bien, ante esta diversidad de objetivos y de estrategias metodológicas cabe preguntarse: ¿cuáles son los objetivos más importantes desde el punto de vista de los propios extensionistas? ¿Cuáles son las estrategias metodológicas que ellos utilizan con mayor frecuencia? Indudablemente, se trata de preguntas relevantes, dado que su respuesta permitirá pasar de la discusión de lo que los académicos dicen que debería hacerse a la descripción empírica de lo que los extensionistas se proponen y hacen en campo. De esta manera, en este trabajo se identifican los objetivos más importantes del trabajo de extensión y las estrategias metodológicas más utilizadas desde el punto de vista de los propios extensionistas rurales. Así, se plantea qué es lo que ellos consideran más importante hacer y cómo lo hacen quienes trabajan en vínculo directo con los productores. 


\section{Metodología}

Para alcanzar el objetivo propuesto en el presente trabajo, se llevó adelante un estudio cuantitativo, transversal y de tipo descriptivo, entre agosto y septiembre del 20I7, centrado en la realización de un cuestionario a extensionistas rurales que trabajan en Argentina. El cuestionario en mención incluyó preguntas sociodemográficas y preguntas de contenido.

\section{Participantes}

Para establecer contacto con los participantes del estudio se obtuvo apoyo de las autoridades del Instituto Nacional de Tecnología Agropecuaria (INTA) y de la Subsecretaría de Agricultura Familiar (SsAF), del antiguo Ministerio de Agroindustria de la Nación. Se eligieron estas dos instituciones por ser los dos organismos de extensión y desarrollo rural más importantes del país.

Para el desarrollo del cuestionario, se utilizó la plataforma SurveyMonkey, a la cual se podía acceder por medio de internet. La primera página contenía un consentimiento informado que debía ser respondido para poder continuar. El INTA generó un listado de 3375 correos de extensionistas pertenecientes a la institución, incluyendo a quienes se habían desempeñado como promotores asesores del programa Cambio Rural, a los cuales se les envió el enlace del cuestionario. La SsAF envió el cuestionario a autoridades provinciales para que lo reenviaran por correo electrónico a sus técnicos de campo. En el momento del estudio, se estimaba que la SsAF contaba con aproximadamente 1000 extensionistas, aunque la importante reducción de personal en dicho periodo hace difícil una estimación ajustada, a lo que se suma la falta de certeza sobre la cantidad de coordinadores provinciales que reenviaron la invitación. $\mathrm{Al}$ atender al procedimiento de toma del cuestionario, la muestra no puede considerarse representativa en un sentido estadístico.

No todas las personas que recibieron los correos y contestaron el cuestionario se encontraban trabajando en ese momento en el INTA o en la SsAF, ya que algunos habían cambiado de institución o se encontraban desempleados. Un total de 593 extensionistas rurales y asesores técnicos que trabajan en Argentina respondieron las preguntas del cuestionario, con un porcentaje del 65,1\% de hombres y 34,9\% de mujeres. El 82,6\% de los que contestaron contaba con título universitario: $50,4 \%$ eran ingenieros agrónomos, $15,2 \%$ veterinarios o ingenieros zootecnistas y $5,7 \%$ profesionales de las ciencias sociales (sociólogos, trabajadores sociales, psicólogos y pedagogos). El resto de los profesionales (II, $3 \%$ ) estaba compuesto por una amplia variedad de disciplinas, incluyendo biólogos, ingenieros en recursos naturales y en alimentos, y licenciados en turismo, en administración y en ciencias ambientales, entre otros. La distribución de las cifras por institución fue $56 \%$ del INTA (incluyendo los programas Cambio Rural y ProHuerta) y i $8,4 \%$ de la SsAF; el resto mencionó a otra institución o prefirió no responder. La edad media de los participantes fue de 43,6 años $(D E=9,75)$ y la experiencia media como extensionistas o asesores técnicos de I2,I años $(D E=8,73)$; en este último caso, se consideró medio año de experiencia cuando los participantes indicaron tener "menos de un año de experiencia", ya que se trataba de un período inespecífico. Respecto de las regiones y las provincias que las conforman, la distribución de respuestas fue la siguiente: Cuyo (La Rioja, Mendoza, San Juan y San Luis), 19,6\%; Noreste (Corrientes, Chaco, Formosa y Misiones), I8\%; Noroeste (Catamarca, Jujuy, Salta, Santiago del Estero y Tucumán), 19,9\%; Pampeana (Buenos Aires, Córdoba, Entre Ríos Santa Fe), 29,7\%; y Patagonia (Chubut, La Pampa, Neuquén, Río Negro, Santa Cruz y Tierra del Fuego), 12,9\%. 


\section{Caracterización de las instituciones de extensión participantes}

La mayor parte de los extensionistas rurales y asesores técnicos que participaron del estudio trabajan en el INTA o en la SsAF. El INTA es una institución pública autárquica, creada en el año 1956, que lleva adelante tanto tareas de investigación agropecuaria como de extensión rural. Todos los territorios rurales del país se encuentran bajo la órbita de unidades o agencias de extensión rural de la institución, las cuales suelen contar con entre tres y cinco extensionistas. Estos proveen asesoramiento técnico gratuito a productores agropecuarios pequeños y medianos, apoyan grupos y organizaciones de productores, entregan insumos como semillas y pollitos a productores de subsistencia, ofrecen capacitaciones y apoyan la coordinación entre instituciones interesadas en el desarrollo rural del país. Dentro de sus líneas de acción, se destaca el programa Cambio Rural, orientado a grupos de medianos productores, y el ProHuerta, destinado a población con necesidades alimentarias insatisfechas o que practica la agricultura de autoconsumo.

La SsAF también se encuentra presente en todo el país. Fue creada en el año 2008, a partir de la institucionalización del Programa Social Agropecuario, el cual inició su funcionamiento en el año 1993, brindando crédito y luego subsidios a grupos y a organizaciones de productores. La SsAF orienta su accionar a la implementación de políticas públicas en el ámbito de la agricultura familiar y el desarrollo rural. Cabe anotar que la falta de lineamientos institucionales claros, unida a la escasez de recursos económicos, ha tenido como resultado que los técnicos de campo de los diferentes territorios lleven adelante una amplia diversidad de acciones, dependiendo del lugar donde se desempeñan y de la disponibilidad de recursos. En general, sus acciones incluyen el relevamiento de información sobre agricultura familiar y el apoyo en la implementación de diferentes proyectos con grupos u organizaciones de productores, en articulación con otras instituciones.

\section{Instrumento}

El cuestionario forma parte de una investigación más amplia, la cual incluye una diversidad de temáticas. En este artículo se indican las preguntas específicamente analizadas. La primera parte del cuestionario incluyó preguntas sociodemográficas, como las informadas previamente al describir a los participantes. Junto a ellas, se procuró identificar cuáles eran los principales objetivos y líneas de acción de los extensionistas, para lo cual se establecieron diez alternativas diferentes; para lograr lo anterior, se realizó un trabajo de revisión bibliográfica que se complementó con resultados empíricos de un estudio previo sobre problemáticas y enfoques de extensión rural en diferentes países latinoamericanos (Landini, 20I6b) y con consultas a expertos en extensión rural de diferentes países.

En términos concretos, se presentaron las diez opciones a los participantes y se pidió que identificaran "los tres objetivos más importantes del trabajo de extensión rural desde su punto de vista”. Las alternativas propuestas fueron:

I. Creación y fortalecimiento de organizaciones de productores.

2. Mejora de la calidad de vida a través del acceso a servicios básicos.

3. Fortalecimiento de las estrategias productivas y de los medios de vida de los agricultores a través de la financiación de (pequeños) proyectos productivos. 
4. Integración de los productores en cadenas comerciales y apoyo a la comercialización en mercados convencionales.

5. Aumento de los conocimientos productivos y comerciales de los agricultores a través de capacitaciones.

6. Modernización productiva para el aumento de la productividad y la rentabilidad.

7. Protección y manejo de recursos naturales.

8. Desarrollo de capacidad emprendedora y empresarial.

9. Resolución de problemas productivos o comerciales planteados por los productores a partir de asesoramiento puntual.

ı. Entrega de información sobre precios o clima para la toma de decisiones.

Al mismo tiempo, se indagó sobre cuáles eran las estrategias metodológicas utilizadas más frecuentemente. Para esto, sobre la base de la revisión bibliográfica realizada, se propusieron como alternativas:

- Trabajo con productores individuales (o productores individuales y su familia).

- Trabajo con grupos u organizaciones de productores.

- Trabajo de articulación o coordinación interinstitucional o inter-actoral.

- Trabajo con medios masivos de comunicación (difusión de técnicas productivas o información a través de medios tales como la radio, teléfono celular, internet, redes sociales, televisión u otro similar).

En concreto, se pidió a los participantes que ordenaran las opciones de aquella que más utilizaban a la que menos utilizaban. Se permitió utilizar el cero en los casos en los cuales una opción no era utilizada en absoluto.

\section{Procedimiento de análisis}

Para el análisis de los resultados obtenidos se utilizó estadística descriptiva. En primer lugar, se describió a nivel porcentual la cantidad de participantes que seleccionaron cada uno de los objetivos de la extensión rural dentro de los "tres objetivos más importantes". Teniendo en cuenta que cada uno de los objetivos constituye una variable nominal dicotómica, con los valores "Seleccionada" y "No seleccionada", se los relacionó con un conjunto de variables de tipo sociodemográfico.

Para el caso de las variables nominales se utilizó Chi Cuadrado ( $\left.\chi^{2}\right)$. Para la variable "Institución” solo se consideró al INTA y a la SsAF, ya que la categoría "Otra institución" no refería a una entidad específica. En el caso de "Título", se diferenció entre "Ingeniero/a Agrónomo/a", "Veterinario/a”, "Ciencias Sociales” y "Otros”. Para el caso de "Título" y "Regiones” (donde los valores de variable son mayores a dos), también se utilizó la Prueba Z, a fin de evaluar no solo si existían diferencias de valoración de objetivos según esas variables a nivel general, sino también entre los diferentes subgrupos. De esta manera, se hizo posible identificar no solo si existen diferencias de valoración de un objetivo de la extensión rural según "Regiones”, sino entre qué regiones específicamente existe tal diferencia. Finalmente, para el caso de las variables ordinales como "Nivel educativo" y "Orden asignado a las diferentes estrategias metodológicas", se utilizó la prueba U de MannWithney. Los valores posibles para "Nivel educativo" fueron: "Ninguno", "Primario", "Secundario", “Terciario (no universitario)”, “Universitario”, “Maestría” y “Doctorado”. Finalmente, para variables de razón se utilizó la prueba T de Student. 


\section{Resultados}

Los resultados obtenidos con el cuestionario se presentan organizados en tablas. A fin de facilitar la lectura, en ellas solo se ofrece información detallada cuando las diferencias identificadas son estadísticamente significativas.

\section{Objetivos del trabajo de extensión rural}

En la tabla i se presenta la cantidad de extensionistas que eligieron los diferentes objetivos entre los tres más importantes de la extensión rural.

TABLA 1.

OBJETIVOS DE LA EXTENSIÓN RURAL MÁS VALORADOS

\begin{tabular}{lrr}
\hline \multicolumn{1}{c}{ Objetivos del trabajo de extensión rural } & n & Promedio \\
\hline $\begin{array}{l}\text { 1. Creación y fortalecimiento de organizaciones de } \\
\text { productores }\end{array}$ & 329 & $56,4 \%$ \\
\hline $\begin{array}{l}\text { 2. Mejora de la calidad de vida a través del acceso a servicios } \\
\text { básicos }\end{array}$ & 207 & $35,5 \%$ \\
\hline $\begin{array}{l}\text { 3. Fortalecimiento de las estrategias productivas y de los } \\
\text { medios de vida de los agricultores a través de la financiación } \\
\text { de (pequeńos) proyectos productivos }\end{array}$ & 206 & $35,3 \%$ \\
\hline $\begin{array}{l}\text { 4. Integración de los productores en cadenas comerciales y } \\
\text { apoyo a la comercialización en mercados convencionales }\end{array}$ & 202 & $34,6 \%$ \\
\hline $\begin{array}{l}\text { 5. Aumento de los conocimientos productivos y comerciales } \\
\text { de los agricultores a través de capacitaciones }\end{array}$ & 198 & $34 \%$ \\
\hline $\begin{array}{l}\text { 6. Modernización productiva para el aumento de la } \\
\text { productividad y la rentabilidad }\end{array}$ & 184 & $31,6 \%$ \\
\hline $\begin{array}{l}\text { 7. Protección y manejo de recursos naturales } \\
\text { 8. Desarrollo de capacidad emprendedora y empresarial }\end{array}$ & 157 & $26,9 \%$ \\
\hline $\begin{array}{l}\text { 9. Resolución de problemas productivos o comerciales } \\
\text { planteados por los productores a partir de asesoramiento } \\
\text { puntual }\end{array}$ & 146 & $25 \%$ \\
\hline $\begin{array}{l}\text { 10. Entrega de información sobre precios o clima para la } \\
\text { toma de decisiones }\end{array}$ & 115 & $19,7 \%$ \\
\hline \begin{tabular}{l} 
Total \\
\hline
\end{tabular} & 583 & $100 \%$ \\
\hline
\end{tabular}

Fuente: elaboración propia

Un primer análisis cuantitativo de los resultados permite dividir los objetivos de la extensión rural en cuatro grupos diferenciados, según su importancia relativa. El primer grupo está conformado por el objetivo más valorado: la "Creación y fortalecimiento de organizaciones de productores". Al ver que este objetivo fue mencionado por más de la mitad de los encuestados y que existe una distancia significativa respecto del segundo, puede afirmarse que se trata de un objetivo central en el contexto argentino. 
En segundo lugar, se observa un grupo de cinco objetivos que poseen escasa diferencia entre sí (los objetivos 2 al 6), los cuales han sido mencionados por entre el 31,6\% y el 35,5\% de los participantes. Luego de estos, se ubica un grupo compuesto por los objetivos 7 al 9 , seleccionados por entre el $19,7 \%$ y el $26,9 \%$ de los extensionistas. Finalmente, el cuarto grupo también está compuesto por un único objetivo: la "Entrega de información sobre precios o clima para la toma de decisiones”, el cual fue mencionado por menos del $\mathrm{I} \%$ de la muestra, lo que sugiere que posee escasa relevancia desde el punto de vista de los encuestados.

\section{Uso de diferentes estrategias metodológicas para el trabajo de extensión rural}

En la tabla 2 se presentan las estrategias metodológicas más utilizadas en el trabajo de extensión rural.

TABLA 2.

USO DE DIFERENTES ESTRATEGIAS EN EL TRABAJO DE EXTENSIÓN RURAL

\begin{tabular}{|c|c|c|c|c|c|}
\hline Tipo de trabajo & $1^{\text {ra }}$ opción & $2^{\text {da }}$ opción & $3^{\text {ra }}$ opción & $4^{\text {ta }}$ opción & No utiliza \\
\hline Individual & $26 \%$ & $29,2 \%$ & $28 \%$ & $13,5 \%$ & $3,4 \%$ \\
\hline Grupal & $59 \%$ & $27,8 \%$ & $8,8 \%$ & $3,2 \%$ & $1,2 \%$ \\
\hline $\begin{array}{l}\text { Coordinación } \\
\text { institucional }\end{array}$ & $17,4 \%$ & $38,4 \%$ & $35,9 \%$ & $6,1 \%$ & $2,2 \%$ \\
\hline $\begin{array}{l}\text { Medios de } \\
\text { comunicación }\end{array}$ & $4 \%$ & $4 \%$ & $18,2 \%$ & $57 \%$ & $16,7 \%$ \\
\hline
\end{tabular}

Fuente: elaboración propia

Los resultados muestran un predominio de estrategias grupales en el trabajo de extensión rural, lo que se hace evidente al comparar las cifras referidas a la primera opción con las del resto. Las estrategias individuales y de coordinación interinstitucional se ubican en segundo lugar, con una muy escasa diferencia entre ellas. Considerando el porcentaje de aquellos que toman estas estrategias como primera opción, se observa un predominio de abordajes individuales, lo que se invierte a nivel de la segunda y tercera opción. Por último, con bastante distancia respecto de las opciones anteriores, aparece el uso de medios masivos de comunicación como estrategia metodológica para la tarea de extensión rural.

\section{Objetivos de la extensión según metodologías usadas y variables sociodemográficas}

A continuación, en las tablas 3 y 4 se estudia la relación entre la valoración dada a los diferentes objetivos, múltiples variables sociodemográficas y las estrategias metodológicas utilizadas por los extensionistas. 
TABLA 3.

OBJETIVOS DE LA EXTENSIÓN RURAL MÁS VALORADOS, SEGÚN DIFERENTES VARIABLES SOCIODEMOGRÁFICAS

\begin{tabular}{|c|c|c|c|c|c|c|c|}
\hline Objetivos & $\begin{array}{l}\text { Sexo } \\
(\%)\end{array}$ & $\begin{array}{l}\text { Edad } \\
M(D E)\end{array}$ & $\begin{array}{l}\text { Experiencia } \\
M(D E)\end{array}$ & $\begin{array}{l}\text { Regiones } \\
(\%)\end{array}$ & $\begin{array}{l}\text { Nivel } \\
\text { educativo } \\
\text { (rango } \\
\text { medio) }\end{array}$ & $\begin{array}{l}\text { Institución } \\
(\%)\end{array}$ & $\begin{array}{l}\text { Título } \\
(\%)\end{array}$ \\
\hline $\begin{array}{l}\text { 1. Creación y } \\
\text { fortalecimiento de } \\
\text { organizaciones de } \\
\text { productores }\end{array}$ & -. & - & $\begin{array}{l}t(579)=-2,30^{*} \\
M_{\mathrm{si} i} 12,8(8,4) \\
\mathrm{M}_{\mathrm{No}}: 11,1(9,1)\end{array}$ & $\begin{array}{l}\chi^{2}(4)=12,4^{*} \\
\text { 'Cuyo: } 66,7 \\
\text { 'Noreste: } 47,2 \\
{ }^{2} \text { Noroeste: } 63,3 \\
\text { 'Pampeana: } 50,9 \\
\text { 'batagonia: } 56,1\end{array}$ & -. & $\begin{array}{l}\chi^{2}(1)=18,6^{* * *} \\
\text { INTA: } 55 \\
\text { SsAF: } 78,5\end{array}$ & 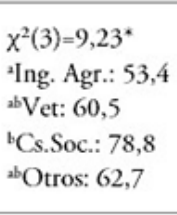 \\
\hline $\begin{array}{l}\text { 2. Mejora de la calidad } \\
\text { de vida a través del } \\
\text { acceso a servicios básicos }\end{array}$ & - & - & - & - & - & $\begin{array}{l}\chi^{2}(1)=3,93^{*} \\
\text { INTA: } 33,3 \\
\text { SsAF: } 43,9\end{array}$ & - \\
\hline $\begin{array}{l}\text { 3. Fortalecimiento de las } \\
\text { estrategias productivas y } \\
\text { de los medios de vida de } \\
\text { los agricultores a través } \\
\text { de la financiación de } \\
\text { (pequeńos) proyectos } \\
\text { productivos }\end{array}$ & $\begin{array}{l}\chi^{2}(1)=20,0 * * \\
H: 29,4 \\
M: 36,5\end{array}$ & - & - & $\begin{array}{l}\chi^{2}(4)=15,8^{* *} \\
\text { 'Cuyo: } 31,6 \\
{ }^{2} \text { Noreste: } 33,3 \\
\text { 'Noroeste: } 51,4 \\
\text { 'Pampeana: } 29,5 \\
\text { 'Patagonia: } 34,1\end{array}$ & $\begin{array}{l}Z=-2,14^{*} \\
\text { No: } 301 \\
\text { Sí: } 275\end{array}$ & $\begin{array}{l}\chi^{2}(1)=34,0 * * \\
\text { INTA: } 31,8 \\
\text { SsAF: } 63,5\end{array}$ & - \\
\hline $\begin{array}{l}\text { 4. Integración de los } \\
\text { productores en cadenas } \\
\text { comerciales y apoyo a la } \\
\text { comercialización en } \\
\text { mercados convencionales }\end{array}$ & - & - & $\begin{array}{l}t(579)=2,56^{*} \\
M_{\mathrm{si}}: 10,8(8,3) \\
\mathrm{M}_{\mathrm{No}}: 12,7(8,9)\end{array}$ & - & - & - & - \\
\hline $\begin{array}{l}\text { 5. Aumento de los } \\
\text { conocimientos } \\
\text { productivos y } \\
\text { comerciales de los } \\
\text { agricultores a través de } \\
\text { capacitaciones }\end{array}$ & - & - & - & - & - & - & - \\
\hline
\end{tabular}

Fuente: elaboración propia

Notas: Notas: ${ }^{*}: p \leq .05 ;{ }^{* *}: p<.01 ;{ }^{* * *}: p<.001$; Sí: la persona seleccionó el objetivo; No: la persona no seleccionó el objetivo; H: hombre; M: Mujer; Ing. Agr.: Ingeniero/a agrónomo/a; Vet: Veterinario/

a; Cs.Soc.: Ciencias sociales; las letras a, b y c en superíndice delante de las regiones y títulos

universitarios (por ej. ${ }^{\mathrm{a}} \mathrm{Cuyo}$ ) hacen referencia a grupos de valores de variable cuyas proporciones difieren significativamente entre sí a un nivel de $p<.05)$. En el caso de Nivel Educativo, se indican los rangos medios. 
TABLA 3.

OBJetivos de LA EXTENSIÓN RURAL MÁS VALORADOS, SEgÚN DIFERENTES VARIABLES SOCIODEMOGRÁFICAS

\begin{tabular}{|c|c|c|c|c|c|c|c|}
\hline Objetivos & $\begin{array}{l}\text { Sexo } \\
(\%)\end{array}$ & $\begin{array}{l}\text { Edad } \\
M(D E)\end{array}$ & $\begin{array}{l}\text { Experiencia } \\
M(D E)\end{array}$ & $\begin{array}{l}\text { Regiones } \\
(\%)\end{array}$ & $\begin{array}{l}\text { Nivel } \\
\text { educativo } \\
\text { (rango } \\
\text { medio) }\end{array}$ & $\begin{array}{l}\text { Institución } \\
(\%)\end{array}$ & $\begin{array}{l}\text { Título } \\
(\%)\end{array}$ \\
\hline $\begin{array}{l}\text { 6. Modernización } \\
\text { productiva para el } \\
\text { aumento de la } \\
\text { productividad y la } \\
\text { rentabilidad }\end{array}$ & $\begin{array}{l}\chi^{2}(1)=29,0^{* * *} \\
H: 39,1 \\
M: 17,3\end{array}$ & - & - & - & - & $\begin{array}{l}\chi^{2}(1)=12,3^{\cdots *} \\
\text { INTA: } 33,6 \\
\text { SsAF: } 15,9\end{array}$ & $\begin{array}{l}\chi^{2}(3)=15,1^{* *} \\
{ }^{4} \text { Ing. Agr.: } 35,8 \\
\text { ahVet: } 32,6 \\
\text { 'Cs.Soc.: } 6,1 \\
\text { 'Otros: } 31,3\end{array}$ \\
\hline $\begin{array}{l}\text { 7. Protección y manejo } \\
\text { de recursos naturales }\end{array}$ &.- & - & $\begin{array}{l}t(240,014)=2,42^{*} \\
\mathrm{M}_{\mathrm{si}}: 13,6(9,9) \\
\mathrm{M}_{\mathrm{Na}}: 11,5(8,2)\end{array}$ & - & - & $\begin{array}{l}\chi^{2}(1)=5,44^{\cdots \cdots} \\
\text { INTA: } 30,3 \\
\text { SsAF: } 18,7\end{array}$ & - \\
\hline $\begin{array}{l}\text { 8. Desarrollo de } \\
\text { capacidad emprendedora } \\
\text { y empresarial }\end{array}$ & - & - & - & - & - & $\begin{array}{l}\chi^{2}(1)=10,2^{* *} \\
\text { INTA: } 28,4 \\
\text { SsAF: } 13,1\end{array}$ & $\begin{array}{l}\chi^{2}(3)=9,53^{*} \\
{ }^{4} \text { Ing. Agr.: } 29,1 \\
\text { 'Vet: } 14 \\
{ }^{\llcorner} \text {Cs.Soc.: } 18,2 \\
{ }^{2} \text { Otros: } 25,7\end{array}$ \\
\hline $\begin{array}{l}\text { 9. Resolución de } \\
\text { problemas productivos o } \\
\text { comerciales planteados } \\
\text { por los productores a } \\
\text { partir de asesoramiento } \\
\text { puntual }\end{array}$ & - & $\begin{array}{l}t(581)=3,11^{* *} \\
\mathrm{M}_{\mathrm{s}:}: 41,0 \\
\mathrm{M}_{\mathrm{N}_{0}: 44,2}\end{array}$ & $\begin{array}{l}t(579)=3,18^{* *} \\
M_{s:}: 9,8 \\
M_{\text {Na: }}: 12,6\end{array}$ & - & - & - & - \\
\hline $\begin{array}{l}\text { 10. Entrega de } \\
\text { información sobre } \\
\text { precios o clima para la } \\
\text { toma de decisiones }\end{array}$ & - & - & - & - & - & - & - \\
\hline
\end{tabular}

Fuente: elaboración propia

Notas Notas: ${ }^{*}: p \leq .05 ;{ }^{* *}: p<.01 ;{ }^{* * *}: p<.001$; Sí: la persona seleccionó el objetivo; No: la persona no seleccionó el objetivo; H: hombre; M: Mujer; Ing. Agr.: Ingeniero/a agrónomo/a; Vet: Veterinario/

a; Cs.Soc.: Ciencias sociales; las letras $a, b$ y $c$ en superíndice delante de las regiones y títulos

universitarios (por ej. ${ }^{\mathrm{a} C u y o}$ ) hacen referencia a grupos de valores de variable cuyas proporciones difieren significativamente entre sí a un nivel de $p<.05)$. En el caso de Nivel Educativo, se indican los rangos medios. 
TABLA 4.

RELACIÓN ENTRE OBJETIVOS PRIORIZADOS Y ESTRATEGIAS METODOLÓGICAS UTILIZADAS

\begin{tabular}{|c|c|c|c|c|}
\hline Objetivos & Individual & Grupal & Institucional & $\begin{array}{l}\text { Medios de } \\
\text { comunicación }\end{array}$ \\
\hline \multirow{3}{*}{ 1. Creación y fortalecimiento de organizaciones de productores } & $U=29002,5^{\cdots}$ & $U=34124,5^{\cdots+}$ & $U=32774,5^{\cdots}$ & \multirow[t]{3}{*}{ 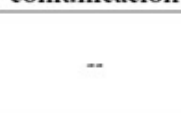 } \\
\hline & Sí: 331 & Sí: 269 & Sí: 265 & \\
\hline & No: 242 & No: 322 & No: 327 & \\
\hline \multirow{2}{*}{$\begin{array}{l}\text { 2. Mejora de la calidad de vida a través del acceso a servicios } \\
\text { básicos }\end{array}$} & $U=34802^{\circ}$ & \multirow[b]{2}{*}{-. } & \multirow[b]{2}{*}{.- } & \multirow[b]{2}{*}{-. } \\
\hline & $\begin{array}{l}\text { Sí: } 312 \\
\text { No: } 281\end{array}$ & & & \\
\hline \multirow{3}{*}{$\begin{array}{l}\text { 3. Fortalecimiento de las estrategias productivas y de los medios } \\
\text { de vida de los agricultores a través de la financiación de } \\
\text { (pequeños) proyectos productivos }\end{array}$} & $U=35145,5^{\circ}$ & & $U=35226^{\circ}$ & \multirow{3}{*}{-} \\
\hline & Sí: 283 & -- & Sí: 206 & \\
\hline & No: 310 & & No: 377 & \\
\hline $\begin{array}{l}\text { 4. Integración de los productores en cadenas comerciales y apoyo } \\
\text { a la comercialización en mercados convencionales }\end{array}$ & -- & -- & -- & -- \\
\hline \multirow{3}{*}{$\begin{array}{l}\text { 5. Aumento de los conocimientos productivos y comerciales de } \\
\text { los agricultores a través de capacitaciones }\end{array}$} & $U=33760^{\circ}$ & $U=34044^{\circ}$ & & \multirow{3}{*}{-} \\
\hline & Sí: 270 & Sí: 313 & -. & \\
\hline & No: 303 & No: 281 & & \\
\hline \multirow{3}{*}{$\begin{array}{l}\text { 6. Modernización productiva para el aumento de la } \\
\text { productividad y la rentabilidad }\end{array}$} & $U=32634,5^{\circ}$ & & & \multirow{3}{*}{-} \\
\hline & Sí: 270 & -. & .- & \\
\hline & No: 302 & & & \\
\hline \multirow{3}{*}{ 7. Protección y manejo de recursos naturales } & \multirow{3}{*}{-- } & \multirow{3}{*}{-- } & \multirow{3}{*}{--} & $U=30183^{\circ}$ \\
\hline & & & & Sí: 271 \\
\hline & & & & No: 300 \\
\hline 8. Desarrollo de capacidad emprendedora y empresarial & - & -- & -- & - \\
\hline \multirow{3}{*}{$\begin{array}{l}\text { 9. Resolución de problemas productivos o comerciales } \\
\text { planteados por los productores a partir de asesoramiento puntual }\end{array}$} & $U=20590^{\ldots}$ & $U=23851,5^{\circ}$ & $U=20819,5^{\ldots}$ & $U=23717^{\circ}$ \\
\hline & Sí: 237 & Si: 319 & Sí: 345 & Si: 320 \\
\hline & No: 305 & No: 285 & No: 279 & No: 285 \\
\hline \multirow{2}{*}{$\begin{array}{l}\text { 10. Entrega de información sobre precios o clima para la toma } \\
\text { de decisiones }\end{array}$} & & & $U=750^{\circ}$ & \multirow[b]{2}{*}{--} \\
\hline & - & -. & $\begin{array}{l}\text { Sí: } 431 \\
\text { No: } 291\end{array}$ & \\
\hline
\end{tabular}

Fuente: elaboración propia

Nota: ${ }^{*}: p \leq .05 ;{ }^{* *}: p<.01 ;{ }^{* * *}: p<.001$; Sí: la persona seleccionó el objetivo; No: la persona no seleccionó el objetivo. Cuando la relación es significativa, se indican los rangos medios.

Los resultados del cuestionario muestran que los objetivos de la extensión rural priorizados por los participantes se encuentran asociados tanto con las estrategias metodológicas que estos utilizan como con diferentes variables sociodemográficas. A continuación, se describen los resultados relativos a cada uno de los objetivos.

\section{Creación y fortalecimiento de organizaciones de productores}

Este objetivo fue mencionado más veces por los extensionistas de la SsAF, en línea con el trabajo realizado desde sus orígenes en el Programa Social Agropecuario con grupos y organizaciones de productores, y por quienes poseen título universitario en ciencias sociales, al estar especialmente formados para abordar estas temáticas. A su vez, quienes destacan este objetivo valoran más el uso de estrategias de trabajo grupal e interinstitucional y menos las de tipo individual, como era de esperarse. Por último, quienes trabajan en las regiones de Cuyo y Noroeste también valoran más la "Creación y fortalecimiento de organizaciones de productores” que quienes lo hacen en las regiones Pampeana y Noreste, sin que sean claras las razones de esta diferencia. 


\section{Mejora de la calidad de vida a través del acceso a servicios básicos}

Se trata de un objetivo más frecuente entre quienes trabajan en la SsAF y quienes priorizan menos el uso de estrategias metodológicas individuales. Respecto de la SsAF, aquí se vuelve a observar una diferencia con el perfil del INTA: la Secretaría se ocupa de aquellas temáticas que conciernen a la agricultura familiar y a su bienestar, mientras que el INTA se especializa en cuestiones más relacionadas con la tecnología agropecuaria, como su nombre lo indica.

\section{Fortalecimiento de las estrategias productivas y de los medios de vida de los agricultores a través de la financiación de (pequeños) proyectos productivos}

Más mujeres que hombres priorizan este objetivo y quienes tienen en promedio un menor nivel educativo, sin que las razones de esto sean claras. También es un objetivo muchísimo más frecuente en quienes trabajan en la SsAF, con respecto a quienes trabajan en el INTA, lo que refuerza nuevamente el perfil diferencial de ambas instituciones. En este caso, posiblemente la historia de la SsAF y su origen en el Programa Social Agropecuario, caracterizado por la entrega de pequeños subsidios para grupos y organizaciones de productores, juegue un papel central en la valorización de este objetivo. Por su parte, en la región Noroeste, los extensionistas destacan más el fortalecimiento de las estrategias productivas de los agricultores a partir del financiamiento de pequeños proyectos productivos, a comparación con las demás regiones. Esto posiblemente se deba a una especificidad regional que no ha sido identificada en este trabajo. Por último, se observa que se trata de un objetivo más valorado por quienes utilizan estrategias metodológicas individuales e interinstitucionales, sin que queden claras las razones de estas diferencias.

\section{Integración de los productores en cadenas comerciales y apoyo a la comercialización en mercados convencionales}

Los resultados de este estudio muestran que quienes mencionan este objetivo con mayor frecuencia tienden a tener menos años de experiencia como extensionistas. Una hipótesis para explicar esta diferencia es que posiblemente los extensionistas más experimentados hayan tenido más tiempo para notar que el apoyo a la integración de los productores en cadenas comerciales convencionales es menos efectiva que lo que podría pensarse a primera vista. 


\section{Aumento de los conocimientos productivos y comerciales de los agricultores a través de capacitaciones}

Se trata de un objetivo más valorado por los extensionistas que priorizan más los abordajes individuales y menos los grupales. Esto es interesante, ya que, si bien la realización de capacitaciones implica la reunión de productores, el reunirlos para capacitarlos parecería estar más relacionado con la lógica de trabajo individual que con uso de metodologías grupales como algo valorado en sí mismo, como cuando se habla de crear o fortalecer organizaciones de productores.

\section{Modernización productiva para el aumento de la productividad y la rentabilidad}

Se trata de un objetivo mencionado con mayor frecuencia por hombres que por mujeres, también por quienes trabajan en el INTA que por quienes lo hacen en la SsAF, y por quienes poseen título de ingenieros agrónomos $(35,8 \%)$ o de veterinarios $(32,6 \%)$, frente a egresados de ciencias sociales (6,1\%). Además, quienes priorizan abordajes metodológicos individuales destacan con más frecuencia la modernización productiva como objetivo de la extensión rural. Estos resultados son especialmente interesantes. No resulta claro por qué los hombres priorizan este objetivo más que las mujeres; tal vez, se debe a que los hombres suelen trabajar más con productores varones y las mujeres con productoras mujeres. En este marco, es posible que la idea de modernización productiva se vincule más a los rubros asociados a los varones, en contraste con las mujeres, más cercanas a la producción de huerta, autoconsumo o de comercialización en ferias, donde la idea de modernización tendría menos relevancia. En cuanto a la mayor valoración de la modernización productiva dentro del INTA, resulta esperable, en tanto se trata de una institución con mayor perfil técnico-productivo, en contraste con la SsAF.

\section{Protección y manejo de recursos naturales}

Este objetivo es priorizado por los extensionistas que poseen más experiencia, por quienes trabajan en el INTA (en contraste con aquellos que lo hacen en la SsAF) y por quienes valoran más el uso de los medios de comunicación masivos en la extensión rural. Respecto de la priorización de este objetivo por quienes poseen más experiencia, esto posiblemente se deba a la falta de formación en sustentabilidad ambiental de los egresados en ciencias agropecuarias, quienes luego reconocen el valor del tema a partir de su experiencia en campo. Con relación a la diferencia a favor de quienes trabajan en el INTA, esta probablemente se vincule con la asociación entre la búsqueda de productividad y de sustentabilidad ambiental que marcó a la institución en el pasado reciente (INTA, 2004). 


\section{Desarrollo de capacidad emprendedora y}

empresarial

Si bien no es un objetivo muy mencionado, se reconoce mucha mayor presencia de extensionistas del INTA, en contraste con aquellos de la SsAF, y entre quienes poseen título de ingenieros agrónomos frente a los veterinarios. En el caso del INTA, no resulta claro el porqué de esta diferencia. En paralelo, sí llama la atención el fuerte contraste observado entre ingenieros agrónomos y veterinarios, tal vez por la mayor dificultad asociada a la comercialización de productores agrícolas (para lo cual se requeriría desarrollar cultura emprendedora y empresarial), en contraste con productos pecuarios (más fáciles de comercializar).

\section{Resolución de problemas productivos o comerciales planteados por los productores a partir de asesoramiento puntual}

Se trata de un objetivo más frecuente en quienes son más jóvenes y poseen menos experiencia, y entre quienes valoran más las metodologías individuales y menos el trabajo grupal, interinstitucional y de medios de comunicación. $\mathrm{Al}$ analizar estos resultados puede pensarse que la resolución de problemas a partir de asesoramiento puntual se relaciona con un modelo más cercano a la formación que reciben los egresados de ciencias agropecuarias, centrado en el asesoramiento y la resolución de problemas a nivel individual, algo que parece ir cambiando con la edad y la experiencia de trabajar en instituciones públicas en extensión rural.

\section{Entrega de información sobre precios o clima para la toma de decisiones}

Este objetivo es más mencionado por quienes utilizan menos estrategias de trabajo interinstitucionales, sin que haya razones claras para explicar esta diferencia. Cabe destacar que la escasa cantidad de personas que priorizaron este objetivo tiende a desbalancear los grupos comparados.

\section{Discusión}

Con el estudio y el análisis de los datos obtenidos del cuestionario se evidenció que el objetivo prioritario de los extensionistas rurales encuestados corresponde a la "Creación y fortalecimiento de organizaciones de productores". En cierto sentido, este resultado es llamativo, en tanto la mayor parte de quienes respondieron al cuestionario y de quienes trabajan en extensión rural son profesionales de las ciencias agropecuarias y biológicas, mientras que pocos 
son egresados en ciencias sociales (Landini y Bianqui, 20I4; Monsalvo Zamora et al., 2017). Pese a esto, también es cierto que existe un importante consenso en la literatura académica en torno al importante papel que cumplen las cooperativas, las organizaciones y los grupos de productores en el marco de la agricultura familiar y el desarrollo rural (Abebaw y Haile, 2013; Boas y Goldey, 2005).

Dentro del grupo de objetivos siguientes (del 2 al 6), mencionados todos ellos por aproximadamente uno de cada tres participantes, llama la atención el de "Mejora de la calidad de vida a través del acceso a servicios básicos”, ubicado en segundo lugar en el listado general. Méndez Sastoque (2006) ha señalado que los extensionistas deben contribuir con la mejora de las condiciones de vida de los agricultores. A su vez, en un estudio cualitativo (Landini, 2016a), extensionistas argentinos describieron como parte de su papel el facilitar el acceso de pobladores rurales a servicios básicos como salud, educación y agua para consumo. Las razones por las cuales los encuestados ubican este objetivo en segundo lugar no resultan claras, aunque pueden relacionarse con que un alto porcentaje de extensionistas de la muestra trabajan (o trabajaron) en el ámbito público. En cualquier caso, este resultado también pone en discusión la relación entre extensión y desarrollo rural (Zwane, 20I2) y lleva a reflexionar si no será que el papel de la extensión rural se ha ido expandiendo progresivamente, en el contexto público argentino, al de agente de desarrollo rural en un sentido amplio.

Dentro del mismo grupo de objetivos (ubicados entre el 2 y el 6) se observa uno genérico, referido al "Aumento de conocimientos productivos y comerciales de los agricultores a través de capacitaciones”, una actividad que se encuentra fuertemente instituida en el marco del trabajo de extensión (Amaro-Rosales y Gortari-Rabiela, 2016). En paralelo, también encontramos la "Integración de los productores en cadenas comerciales y apoyo a la comercialización en mercados convencionales”, una propuesta que conserva su relevancia pese al creciente interés generado por los circuitos cortos de comercialización (Craviotti y Soleno Wilches, 2015). Asimismo, dentro del mismo grupo se ubica el "Fortalecimiento de las estrategias productivas y de los medios de vida de los agricultores a través de la financiación de (pequeños) proyectos productivos”, lo que se enmarca claramente en la existencia de diferentes proyectos (públicos o apoyados por las $\mathrm{ONG}$ ) que se orientan a la financiación de pequeñas iniciativas para superar las limitaciones de capital que suelen enfrentar los agricultores familiares (Gaitán y Pachón, 20ro).

El último de los objetivos de este grupo (ubicado en sexto lugar) corresponde a la "Modernización productiva para el aumento de la productividad y la rentabilidad”. Llama la atención que la propuesta no haya sido mencionada por más participantes, en tanto que este corresponde al objetivo más tradicional de la extensión rural (Rogers, 1962), lo que contrasta con el primer lugar en que se ubica la "Creación y fortalecimiento de organizaciones de productores".

En cuanto a los objetivos ubicados del séptimo al noveno lugar, se destaca la escasa valoración recibida por la "Protección y manejo de los recursos naturales", teniendo en cuenta la importancia de la sustentabilidad ambiental en el desarrollo rural y la función clave que juega la extensión pública en el manejo sustentable de los recursos naturales (Rivera, 20II). Por su parte, el “Desarrollo de capacidad emprendedora y empresarial” también fue mencionado por una cantidad reducida de extensionistas, lo que sugiere que la propuesta de convertir a los productores en empresarios agrícolas no constituye una línea de acción priorizada en Argentina, posiblemente por reconocerse que la agricultura familiar se rige por imperativos que no se identifican con la lógica del emprendedor capitalista (Paz, 2017). En cuando a la "Resolución de problemas productivos o comerciales planteados por los productores a partir de asesoramiento puntual”, una propuesta característica del asesoramiento técnico privado, se observa que fue de los objetivos menos seleccionados en el estudio, lo que resulta consistente con una muestra donde predominan extensionistas del sistema público.

Por último, llama poderosamente la atención que solo el o,9\% de la muestra (cinco personas) hayan seleccionado como objetivo de la extensión rural la "Entrega de información sobre precios o clima para la toma de decisiones", teniendo en cuenta la relevancia que tiene la información a la hora de decidir (Christoplos, 20ı0). Si bien es posible que este tipo de tareas estén centralizadas y sean llevadas a cabo por pocas personas en las instituciones de extensión, 
o que los extensionistas valoren este objetivo pero no dentro de los tres primeros, es probable que los sistemas de extensión estén perdiendo oportunidades de apoyar a los productores al no aportar información relevante para la toma de decisiones.

En este estudio se evidenció el uso predominante de estrategias metodológicas grupales en el trabajo de extensión por sobre alternativas individuales, de coordinación institucional y de uso de medios de comunicación. Lo anterior va claramente en línea con el menor costo de la asistencia por productor cuando se trabaja con formatos grupales, en comparación con el uso de metodologías individuales (Garforth, 1994; Ndoro et al., 20I4). No obstante, la ubicación en el primer lugar del objetivo de "Creación y fortalecimiento de organizaciones de productores" también evidencia el valor intrínseco de las estrategias grupales, por ejemplo, para apoyar el intercambio de experiencias entre productores (Samuel et al., 20I2), o para facilitar el apoyo mutuo de diferentes maneras (Landini et al., 20I7).

Las metodologías individuales o de trabajo de coordinación interinstitucional se ubicaron en segundo y tercer lugar respectivamente, con gran diferencia respecto de las metodologías grupales, pero con escasa diferencia entre ellas. Cabe destacar que las metodologías de extensión individuales son más costosas por productor asistido, aunque también tienen mayor potencial en la resolución de problemas complejos, en el marco de situaciones específicas que difícilmente podrían abordarse de forma grupal (Eberle y Shroyer, 2000). En paralelo, el trabajo de articulación interinstitucional suele relacionarse con ciertos papeles dentro de las organizaciones de extensión (por ejemplo, jefes de agencias o unidades de extensión rural), pero también con la adopción de un enfoque territorial para pensar la extensión y el desarrollo rural (Torrado Porto y Catullo, 2017).

En cuarto lugar, se ubicó el uso de medios masivos de comunicación para la tarea de extensión. En este contexto, cabe preguntarse seriamente si los medios de comunicación masivos, como serían la radio, teléfono celular, internet o redes sociales, están siendo subutilizados por los extensionistas rurales argentinos, lo que implicaría una pérdida de oportunidades para impulsar procesos de desarrollo.

Los resultados relativos a la priorización de objetivos y a las estrategias metodológicas más utilizadas invitan a reflexionar sobre la formación de los extensionistas. Tanto en Argentina como en América Latina, la mayor parte de los extensionistas son técnicos o profesionales de las ciencias agrarias, principalmente ingenieros agrónomos (Landini y Bianqui, 2014). Sin embargo, los extensionistas participantes de este estudio identificaron como objetivo más importante la "Creación y fortalecimiento de organizaciones de productores” e informaron utilizar con mucha mayor frecuencia estrategias metodológicas grupales frente a individuales. Esto evidencia un contraste entre la formación de base de los extensionistas y un componente relevante en su práctica (Alves y Saquet, 20r4; Selis et al., 2013), lo que se fortalece incluso al observar que el objetivo priorizado en segundo lugar es la "Mejora de la calidad de vida a través del acceso a servicios básicos” (como salud, educación y agua potable). Es cierto que varios objetivos valorizados poseen un fuerte componente técnico-productivo, como el "Fortalecimiento de las estrategias productivas y de los medios de vida de los agricultores a través de la financiación de (pequeños) proyectos productivos" y la "Modernización productiva para el aumento de la productividad y la rentabilidad”. No obstante, el perfil de objetivos y metodologías descripto pone en cuestión el fuerte predominio de profesionales de las ciencias agrarias en el papel de extensionistas, lo cual obliga a reconocer la necesidad de una mayor diversidad que incluya tanto profesionales de las ciencias sociales como otros especializados en comercialización y mercados. A la vez, atendiendo al carácter transversal de las metodologías grupales en el marco de la extensión, se hace evidente la importancia de considerarla como un área prioritaria de formación en servicio.

En este estudio también se analizó el vínculo entre los objetivos priorizados, las metodologías utilizadas y diversas variables sociodemográficas. Los resultados alcanzados fueron diversos. A nivel general, puede decirse que la institución de pertenencia incide fuertemente en los objetivos que priorizan los extensionistas a nivel personal, lo que demuestra que las instituciones tienen la capacidad de moldear las intencionalidades (es decir, los objetivos) de sus integrantes. De hecho, seis de los diez objetivos considerados fueron valorados de manera diferente según la institución. No obstante, este estudio también muestra que existen diferencias relevantes a 
nivel personal al momento de priorizar objetivos, en particular, la experiencia como extensionistas y la formación académica, además del sexo, el nivel educativo y la edad. Estos resultados confirman el supuesto de que las instituciones inciden en los objetivos y metodologías utilizadas por los extensionistas, pero no los determinan, existiendo importantes diferencias interindividuales relativas a otras variables.

Como limitaciones de este estudio cabe señalar que los resultados obtenidos están influidos por la lista específica de objetivos utilizados. Esta lista fue construida a partir de una revisión bibliográfica amplia, complementada con una consulta a expertos y con los resultados de un estudio empírico previo, por lo cual confiamos que es suficientemente diversa. No obstante, la inclusión de objetivos diferentes en el cuestionario (o una modificación en su redacción) podría generar resultados potencialmente diferentes.

A la vez, cabe recordar que los participantes del estudio respondieron voluntariamente al cuestionario y no fueron seleccionados de manera aleatoria, lo que implica que la muestra utilizada no debe ser considerada estadísticamente representativa. De esta forma, los resultados no pueden ser generalizados a toda la población y solo aplican a la muestra, que además se caracteriza por estar fuertemente asociada a extensionistas que se desempeñan en el sector público.

Como línea futura de investigación, se destaca el interés de observar en terreno de qué manera extensionistas con diferentes objetivos configuran sus acciones, a fin de comprender cómo diferentes intencionalidades se transforman en prácticas diferenciadas y cómo indicen en ellas las instituciones a las que se pertenece.

\section{Conclusiones}

En el presente artículo se analizaron los objetivos más importantes del trabajo de extensión rural, desde el punto de vista de extensionistas rurales argentinos, así como las estrategias metodológicas que estos utilizan con más frecuencia. Los resultados contribuyen a comprender qué es lo que los extensionistas se proponen con su labor y cómo buscan conseguirlo, teniendo en cuenta que las instituciones de extensión suelen establecer formatos de trabajo o actividades a realizar, pero no las intencionalidades con las cuales estas deben llevarse adelante.

De los diez objetivos propuestos, la "Creación y fortalecimiento de organizaciones de productores" fue considerado el más importante, con diferencias cuantitativas relevantes respecto del resto. En paralelo, llamó la atención el sexto lugar en que se ubicó el objetivo de "Modernización productiva para el aumento de la productividad y la rentabilidad”, pues se intuía que este iba a estar ubicado en una posición más elevada, incluso en primer lugar. Al mismo tiempo, genera cierta preocupación la ubicación en séptimo lugar del objetivo de "Protección y manejo de recursos naturales", atendiendo al papel clave que tiene la extensión pública en el impulso de procesos de desarrollo sustentable. Por último, fueron muy pocos los extensionistas que valoraron la "Entrega de información sobre precios o clima para la toma de decisiones" a los productores, lo que invita a reflexionar si realmente su utilidad es tan baja o si constituye una línea de acción subutilizada.

En cuanto a las metodologías de extensión más utilizadas, se observó un claro predominio de estrategias grupales, ubicándose en segundo lugar las individuales y en tercero las de articulación o coordinación interinstitucional. En términos generales, puede afirmarse que los entrevistados valoran intrínsecamente las metodologías grupales, con una creciente importancia dada a la articulación interinstitucional, en el marco de perspectivas de desarrollo pensadas a partir de la idea de territorio. Por último, el escaso uso de medios masivos de comunicación para el trabajo de extensión invita a reflexionar sobre su posible aprovechamiento.

Los resultados también evidenciaron la incidencia de distintas variables en los objetivos que se proponen y en las metodologías que utilizan los extensionistas. Como era previsible, este estudio muestra la incidencia de los marcos institucionales en las prácticas de los extensionistas. No obstante, también evidencia la existencia de perfiles 
diferenciales, basados fundamentalmente en diferencias a nivel de formación y experiencia, aunque también en relación con el sexo, el nivel educativo y la edad de los extensionistas.

\section{Agradecimientos}

Los autores agradecen a las autoridades respectivas del Instituto Nacional de Tecnología Agropecuaria (INTA) y de la antigua Subsecretaría de Agricultura Familiar (actual Subsecretaría Agricultura Familiar y Desarrollo Territorial) por el apoyo brindado para la realización de este estudio.

El presente proyecto de investigación fue financiado por la Universidad de la Cuenca del Plata, en el marco del proyecto "Evaluación de las concepciones de extensión rural de extensionistas de diferentes países" (2016-2019). A la vez, contó con el apoyo del Global Forum for Rural Advisory Services, en el marco de un convenio de colaboración entre ambas instituciones.

\section{Referencias}

Abdu-Raheem, K. (20I4). Exploring the role of agricultural extension in promoting biodiversity conservation in KwaZulu-Natal Province, South Africa. Agroecology and Sustainable Food Systems, 38(9), 1015-1032. https://doi .org/10.1080/21683565.2014.899283

Abebaw, D., y Haile, M. (2013). The impact of cooperatives on agricultural technology adoption: Empirical evidence from Ethiopia. Food Policy, 38, 82-91. https://doi.org/10.1016/j.foodpol.2012.10.003

Acunzo, M., Pafumi, M., Torres, C., y Tirol, M. (2016). Manual de comunicación para el desarrollo rural. FAO.

Alves, A., y Saquet, M. (20I4). La reproducción de las cooperativas de agricultura familiar y economía solidaria: el caso de la unión nacional de cooperativas de la agricultura familiar y economía solidaria (UNICAFES). Perfil de Coyntura Económica, 23, I25-I44. https://www.redalyc.org/pdf/861/86132269007.pdf

Amaro-Rosales, M., y Gortari-Rabiela, R. (2016). Políticas de transferencia tecnológica e innovación en el sector agrícola mexicano. Agricultura, Sociedad y Desarrollo, 13(3), 449-47I. https://doi.org/10.22231/asyd.viziz.406

Ardila, J. (2010). Extensión rural para el desarrollo de la agricultura y la seguridad alimentaria. Aspectos conceptuales, situación y una visión de futuro. IICA.

Boas, A., y Goldey, P. (2005). A comparison on farmers' participation in farmers' organizations and implications for rural extension in Minas Gerais. Organizaçôes Rurais and Agroindustriais, 7(3), 259-270. https://doi.org/ı .22004 /ag.econ.43922

Christoplos, I. (2010). Cómo movilizar el potencial de la extensión agraria y rural. FAO.

Craviotti, C., y Soleno Wilches, R. (2015). Circuitos cortos de comercialización agroalimentaria: un acercamiento desde la agricultura familiar diversificada en Argentina. Mundo Agrario, I6(33). http://www.scielo.org.ar/p $\mathrm{df} /$ magr/vi6n33/vi6n3zaor.pdf

Eberle, W., y Shroyer, J. (2000). Are traditional extension methodologies extinct or just endangered? Journal of Natural Resources and Life Sciences Education, 29, 135-I40. https://doi.org/I0.2134/jnrlse.2000.0135

Freire, P. (1973). ¿¿Comunicación o extensión? La concientización en el medio rural. Siglo XXI.

Gaitán, C., y Pachón, F. (2010). Causas para la adopción de tecnologías para la renovación de cafetales - Caso El Colegio (Cundinamarca). Agronomía Colombiana, 28(2), 329-336. http://www.scielo.org.co/pdf/agc/v28n2/v2 8nza22.pdf 
Galindo González, G., Pérez Trujillo, H., López Mendiola, C., y Robles Martínez, A. (200I). Estrategia de comunicación en el medio rural zacatecano para transferir innovaciones agrícolas. Terra Latinoamericana, I9(4), 393-398. https://www.redalyc.org/pdf/573/57319412.pdf

Garforth, G. (1994). Rural people's organizations and agricultural extension in the Upper North of Thailand: Who benefits? Journal of International Development, 6(6), 707-720. https://doi.org/10.1002/jid.3380060604

González Tena, P. Rendón Medel, R., Sangerman-Jarquín, D. Cruz Castillo, J., y Díaz José, J. (2015). Extensionismo agrícola en el uso de tecnologías de la información y comunicación (TIC) en Chiapas y Oaxaca. Revista Mexicana de Ciencias Agrícolas, 6(I), 175-186. https://doi.org/10.29312/remexca.v6ir.748

Instituto Nacional de Tecnología Agropecuaria [INTA]. (2004). El INTA que queremos: Plan Estratégico Institucional 2005-2015. INTA.

Kingiri, A., y Nderitu, S. (20I4). Assessment of extension and advisory methods and approaches to reach rural women (examples from Kenya). Modernizing Extension and Advisory Services (MEAS).

Landini, F. (2016a). Enfoques y prácticas de extensión rural públicas en el noreste argentino. Revista de Economia e Sociologia Rural, 54(I), 167-I86. https://doi.org/10.1590/1234-56781806-9479005401009

Landini, F. (2016b). Problemas de la extensión rural en América Latina. Perfiles Latinoamericanos, 24(47), 47-68. htt ps://doi.org/10.18504/pl2447-005-2016

Landini, F., Vargas, G., Bianqui, V., Mathot, M., y Martinez, M. (2017). Contributions to group work and to the management of collective processes in extension and rural development. Journal of Rural Studies, 56, I43-155. https://doi.org/10.1016/j.jrurstud.2017.09.014

Landini, F., y Bianqui, V. (2014). Socio-demographic profile of different samples of Latin American rural extensionists. Ciência Rural, 44(3), 575-581. http://dx.doi.org/10.1590/Soro3-84782014000300030

Méndez Sastoque, M. (2006). Los retos de la extensión ante una nueva y cambiante noción de lo rural. Revista Facultad Nacional de Agronomía Medellín, 59(2), 3407-3423.http://www.scielo.org.co/pdf/rfnam/v59n2/aorv59 n2.pdf

Monsalvo Zamora, A., Jiménez Velázquez, M., García Cué, J., Sangerman-Jarquín, D., Martínez Saldaña, T., y Pimentel Equihua, J. (2017). Caracterización del perfil del extensionista rural en la zona oriente del Estado de México. Revista Mexicana de Ciencias Agrícolas, 8(3), 503-515. https://doi.org/10.29312/remexca.v8i3.27

Ndoro, J., Mudhara, M., y Chimonyo, M. (2014). Livestock extension programmes participation and impact on smallholder cattle productivity in Kwazulu-Natal: A propensity score matching approach. South African Journal of Agricultural Extension, 42(2), 62-80. https://doi.org/10.4314/SAJAE.V 42I2

Paz, R. (2017). Las grietas de los agronegocios y los imperativos de la agricultura familiar: hacia una perspectiva conceptual. Revista Latinoamericana de Estudios Rurales, 2(3), 39-63. http://www.fao.org/family-farming/detai l/es/c/903739/

Rivera, W. (20II). Public sector agricultural extension system reform and the challenges ahead. Journal of Agricultural Education and Extension, I7(2), I65-180. https://doi.org/I0.1080/1389224X.2011.544457

Rogers, E. (1962). Diffusion of innovations. The Free Press.

Rossi, V. (20II). Aportes metodológicos para el asesoramiento técnico y la extensión rural. Revista Cangué, 2(3I), 5I-60. http://www.eemac.edu.uy/cangue/joomdocs/cangueo3I_rossi.pdf

Samuel, Z., Kpieta, B., y Godfred, S. (2012). Promoting community-based extension agents as an alternative approach to formal agricultural extension service delivery in Northern Ghana. Asian Journal of Agriculture and Rural Development, 2(I), 76-95. https://doi.org/I0.22004/ag.econ.197944 
Schejtman, A., y Berdegué, J. (2004). Desarrollo territorial rural. Debates y temas rurales. Centro Latinoamericano para el Desarrollo Rural. https://www.rimisp.org/wp-content/files_mf/r363093392schejtman_y_berdegue2004_de sarrollo_territorial_rural_5_rimisp_CArdumen.pdf

Selis, D., Velarde, I., Garat, J., García, B., Otero, J., Villulla, D., y Vértiz, P. (2013). Extensión rural en tiempos de desarrollo territorial local: un análisis comparado en la región ampliada de La Plata. ReD+ER. I(2), 64-89. h ttps://doi.org/10.14409/r.vii2.4468

Stevens, J. (2017). Is agricultural extension positioned to promote agripreneurship in South Africa? South African Journal of Agricultural Extension 45(2), 86-94. http://dx.doi.org/10.17159/2413-3221/2017/v45nia437

Sulaiman, R., y Davis, K. (20I2, noviembre). El “Nuevo Extensionismo”: funciones, estrategias y capacidades para reducir el hambre y la pobreza. En el Global Forum for Rural Advisory Services. Lindau, Suiza.

Torrado Porto, R., y Catullo, J. (2017). Extensión rural y enfoque territorial: aprendiendo en la acción con otros. Revista de la Facultad de Agronomía, I16(3), 19-27. https://revistas.unlp.edu.ar/revagro/article/view/6140/5089

Zwane, E. (2012). Does extension have a role to play in rural development? South African Journal of Agricultural Extension, 4o(I), i6-24. http://www.scielo.org.za/pdf/sajae/v4onı/o2.pdf

\section{Notas}

* $\quad$ Artículo de investigación

CC BY 